OPEN ACCESS

Edited by:

Friedhelm C. Hummel, Institut Fédéral Suisse de Technologie, Switzerland

Reviewed by:

Tiffany Corbet,

École Polytechnique Fédérale de Lausanne, Switzerland Antonio Suppa,

Sapienza Università di Roma, Italy

*Correspondence:

Tanvi Bhatt

tbhatt6@uic.edu

Received: 31 October 2017

Accepted: 16 August 2018 Published: 26 September 2018

Citation:

Bhatt T, Patel P, Dusane S, DelDonno SR and Langenecker SA (2018) Neural Mechanisms Involved in Mental Imagery of Slip-Perturbation While Walking: A Preliminary fMR

Front. Behav. Neurosci. 12:203 doi: 10.3389/fnbeh.2018.00203

\section{Neural Mechanisms Involved in Mental Imagery of Slip-Perturbation While Walking: A Preliminary fMRI Study}

\author{
Tanvi Bhatt ${ }^{1 *}$, Prakruti Patel ${ }^{1}$, Shamali Dusane ${ }^{1}$, Sophie R. DelDonno ${ }^{2}$ and \\ Scott A. Langenecker ${ }^{2}$
}

'Department of Physical Therapy, College of Applied Health Sciences, Chicago, IL, United States, ${ }^{2}$ Department of Psychiatry, College of Medicine, University of Illinois at Chicago, Chicago, IL, United States

Background: Behavioral evidence for cortical involvement in reactive balance control in response to environmental perturbation is established, however, the neural correlates are not known. This study aimed to examine the neural mechanisms involved in reactive balance control for recovery from slip-like perturbations using mental imagery and to evaluate the difference in activation patterns between imagined and observed slipping.

Methods: Ten healthy young participants after an exposure to regular walking and slipperturbation trial on a treadmill, performed mental imagery and observation tasks in the MR scanner. Participants received verbal instructions to imagine walking (IW), observe walking (OW), imagine slipping (IS) and observe slipping (OS) while walking.

Results: Analysis using general linear model showed increased activation during IS versus IW condition in precentral gyrus, middle frontal gyrus, superior, middle and transverse temporal gyrus, parahippocampal gyrus, cingulate gyrus, insula, pulvinar nucleus of the thalamus, pons, anterior and posterior cerebellar lobes. During IS versus OS condition, there was additional activation in parahippocampus, cingulate gyrus, inferior parietal lobule, superior temporal, middle and inferior frontal gyrus.

Conclusion: The findings of the current study support involvement of higher cortical and subcortical structures in reactive balance control. Greater activation during slipping could be attributed to the complexity of the sensorimotor task and increased demands to maintain postural stability during slipping as compared with regular walking. Furthermore, our findings suggest that mental imagery of slipping recruited greater neural substrates rather than observation of slipping, possibly due to increased sensory, cognitive and perceptual processing demands.

New and Noteworthy: The behavioral factors contributing to falls from external perturbations while walking are better understood than neural mechanisms underlying the behavioral response. This study examines the neural activation pattern associated with reactive balance control during slip-like perturbations while walking through an $\mathrm{AMRI}$ 
paradigm. This study identified specific neural mechanisms involved in complex postural movements during sudden perturbations, to particularly determine the role of cortical structures in reactive balance control. It further highlights the specific differences in neural structures involved in regular unperturbed versus perturbed walking.

Keywords: reactive balance control, locomotion, falls, imaging, neural activation

\section{INTRODUCTION}

About 60 percent of outdoor falls sustained by community-living older adults are due to environmental perturbations in the form of a slip or a trip (Luukinen et al., 2000). Behavioral studies exposing older adults to perturbations during walking have established several biomechanical factors such as deficient limb support, inefficient compensatory stepping, reduced stability and lower hip height as predictors of falls (Pavol et al., 2004; Pavol and Pai, 2007; Yang et al., 2009, 2011). While biomechanical causes of falls are better understood, the exact neural mechanisms and substrates involved in recovering balance from sudden falls, in other words for reactive balance control, are still not known. Considering that falls are a significant health concern in the aging and neurological population, understanding the specific neural mechanisms involved in falls occurring from sudden balance loss could assist in developing targeted interventions (Luukinen et al., 2000).

Humans rely on two main strategies for balance control and fall prevention: proactive (occurring before balance disturbance) and reactive (occurring after balance disturbance) control strategies. Proactive strategies are employed to enhance stability to prevent and compensate for a loss of balance even before it occurs (Tang et al., 1998; Ferber et al., 2002; Horak, 2006; Shumway-Cook and Woollacott, 2007). For proactive adjustments, the nervous system has adequate time to predict the upcoming balance requirements and make necessary adjustments to minimize the intensity of balance loss or prevent it altogether (Massion, 1994; Aruin and Latash, 1995). A good example is early detection and avoidance of an upcoming obstacle in the walking path (Woollacott and Tang, 1997). Proactive adjustments during locomotion and functional tasks seem to involve cortical and sub-cortical structures of the CNS for planning and execution of an appropriate motor response in anticipation of a perceived threat to balance (Armstrong and Drew, 1985; Drew, 1988; Beloozerova and Sirota, 1993; Petersen et al., 2012). Indeed, a few electrophysiological studies in humans and cats have confirmed the involvement of motor cortex in regulating accurate muscle activity and modulating step cycle when a change in the environment or obstacle in the path is perceived, (Armstrong and Drew, 1985; Drew, 1988; Beloozerova and Sirota, 1993; Petersen et al., 2012).

Unlike proactive adjustments, reactive balance strategies are the key defense mechanisms to prevent a fall after a sudden unexpected perturbation. It involves compensatory postural adjustments initiated by feedback processes (Maki and McIlroy, 1997). Overall, it is proposed that the CNS uses an internal representation of body's mechanics to predict and identify loss of balance. Often, large postural responses displacing center of mass (COM) outside of the base of support (BOS) require changein-support i.e., stepping to prevent a fall (Maki and McIlroy, 1997; Pai and Bhatt, 2007). Several electrophysiological studies in animals and humans support that polysynaptic spinal cord reflexes (short- and long-latency) are responsible for generating the postural response to correct or restore balance during minor disturbances that do not truly pose a threat to the person (Forssberg et al., 1975; Berger et al., 1984; Dietz et al., 1986; Diener et al., 1988; Allum and Honegger, 1992; Zehr et al., 1997). Reactive responses to restore balance from a large disturbance on the other hand, are usually controlled by supra-spinal structures in the brainstem and its descending pathways (longloop reflexes)(Forssberg et al., 1975; Nashner, 1976, 1980; Eng et al., 1994), with some earlier evidence suggesting modulation from cortical structures (Forssberg et al., 1975). Specifically, cerebral cortex perhaps modifies postural response through cerebellar-cortical loop which helps in using prior experience and basal ganglia-cortical connections which incorporates sensory information based on the current context (Graydon et al., 2005; Jacobs and Horak, 2007).

Several studies have attempted to examine neural mechanisms of compensatory postural responses during stance perturbations using techniques such as electroencephalography (EEG) and functional near infrared spectroscopy (fNIRS) (Quant et al., 2004; Adkin et al., 2006; Mochizuki et al., 2009). However, neural substrates involved in maintaining/recovering balance during locomotor tasks have not been sufficiently examined. This could be partly related to the technical challenges that present with using functional neuroimaging such as fNIRS and EEG measures with walking in the form of either low spatial resolution or noise and artifact (Sipp et al., 2013). Although direct evidence via functional magnetic resonance imaging (fMRI) is difficult to obtain, mental imagery has been widely used to examine locomotor-balance tasks that cannot be performed in the MRI scanner such as walking (Jahn et al., 2004, 2008; van der Meulen et al., 2014) or obstacle crossing (Wang et al., 2009). Use of mental imagery is based on the premise that overlapping neural networks are engaged in both mental imagery and overt movements (Decety and Jeannerod, 1995; Porro et al., 1996). Similarly, action observation has also been used to elicit simulation of motor tasks during neuroimaging - especially gross whole body tasks that cannot be performed in the scanner (Dalla Volta et al., 2015). Action observation is believed to stimulate the neural presentation of the movement being observed through the mirror neuron system (Buccino, 2014). While there are a few studies directly comparing the cortical activation between action observation and actual movement performance, there is only one 
study directly comparing differences in neural activation during observed versus imagined walking (Iseki et al., 2008).

Mental imagery and action observation are increasingly used as rehabilitation tools for inducing motor learning (Morganti et al., 2003; Franceschini et al., 2010; Zhu et al., 2015; Caligiore et al., 2017). Only recently these techniques have been implemented for physical rehabilitation in clinical populations (Ietswaart et al., 2011; Hong et al., 2012; Garcia Carrasco and Aboitiz Cantalapiedra, 2016; Patel et al., 2017); however, they have been widely used to improve performance in sports (Mulder, 2007). It is believed that motor imagery and action observation can prime the CNS to enhance motor learning (fewer repetitions or lesser time) when performed before the actual motor task (Edwards et al., 2003; Hardwick and Edwards, 2011; Saimpont et al., 2013). Such learning mechanisms might be beneficial for frail or neurological populations for whom tolerability of training dosage or safety might be a concern. In the context of reactive balance control, a previous study has examined if observational learning could substitute for motor learning induced via slip perturbation-training for lowering fall-risk (Bhatt and Pai, 2008). The study did demonstrate evidence of improved postural stability with observational training although not to the same extent as that induced by actual motor training. It remains to be determined if mental imagery could have produced a similar or better effect than observational learning, especially due to animation of sensory systems when performing mental imagery with eyes closed (Marx et al., 2003).

Due to lack of literature comparing motor imagery and action observation directly, there is limited understanding of the neural structures underlying these paradigms. In the present pilot study, a novel paradigm using imagined slipping during walking was used to identify neural correlates of modulating locomotor-balance control during environmental perturbations. Our primary objective was to identify the neural networks involved in reactive balance control in response to large sliplike perturbations during walking as compared with regular walking through metal imagery. Considering that slipping while walking demands greater postural control to regain balance, we postulate that during imagined slipping while walking there will be higher activation in the frontal, parietal regions and cerebellum as compared with imagined walking. We also aimed to examine whether or not imagination of slipping while walking results in activation of different brain areas as compared to observation of the same movement. This understanding of neural correlates involved in falls may provide insights for designing observational learning or mental imagery paradigms for fall prevention, especially for populations that might not be able to directly withstand the actual task-specific perturbation training.

\section{MATERIALS AND METHODS}

\section{Subjects}

Ten young healthy adults $(26.90 \pm 4.25$ years $)$ participated in the study after providing an informed consent approved by the Institutional Review Board. The subjects were screened for any systemic disorders and fMRI safety, and had normal vision and hearing. All the subjects then performed the experimental tasks outside and then in the MRI scanner as described below.

\section{Walking and Slipping on Treadmill}

All subjects were initially exposed to walking on the treadmill at a self-selected pace and then were exposed to a single slip-like perturbation with an acceleration of $12.00 \mathrm{~m} / \mathrm{s}^{2}$, displacement $0.25 \mathrm{~m}$ and duration of $0.25 \mathrm{~s}$. For these tasks, subject stood on the ActiveStep treadmill (Simbex, $\mathrm{NH}$ ) with a comfortable stance. A harness suspended from the overhead arch was donned to prevent individuals from falling (Figure 1A). The treadmill belt speed was increased slowly until the individual perceived the speed to be similar to his/her regular walking speed. At this speed, the individual walked for a minute. Following regular walking, the individual performed another walking trial. During this trial individuals were warned that they may experience a sliplike perturbation after a few steps of walking, however, they were unaware of the exact instance of slip occurrence. Upon a sliplike perturbation, individuals were asked to execute their natural response to recover balance and prevent themselves from falling. The subjects were exposed to the treadmill slip before the fMRI experiment to provide them with an experience of how a reallife slip would feel like, especially for those people who might not have experienced a slip in recent times. Such experience could also aid in reducing within-subject variance of the observation or imagination tasks, and hence, enhance ability of the subjects to better relate to the conditions.

\section{Observed and Imagined fMRI Conditions}

After the initial walking and slip exposure, and prior to the MRI scans, subjects underwent a practice session involving motor imagery and observation tasks. The motor imagery task comprised of (a) imagining themselves walking on the treadmill (IW) and (b) imagining themselves slipping on the treadmill while walking, similar to that experienced during initial exposure (IS). Similarly, the observation tasks consisted of (a) observing a video of another person walking on the treadmill (OW) and (b) observing a video of another person slipping while walking on the treadmill (OS) (Figure 1A). During the experiments, the subjects lied down supine in the scanner and the stimuli were projected on the screen in front of them through a mirror with an angle of $9^{\circ}$ from the vertical. For the imagined conditions, subjects received clear instructions to close their eyes and that they should imagine themselves slipping where the leg suddenly slides/moves forward with respect to their body, similar to what they experienced on the treadmill. Such experimental design was purposefully chosen based on the practical realities of the research question, for example if eyes are open for imagined condition this would not be representative of a real life task (challenge to ecological validity). Furthermore, eyes closed during imagination has shown increased cortical activation of various sensory systems as the subjects recall their sensory experience from the perception of the actual activity (Marx et al., 2003). For the observed condition, subjects were instructed to observe the video of a person either walking on the treadmill or slipping while walking on the treadmill, a situation similar to what they had experienced in the laboratory. To ensure subjects engaged themselves during 
A

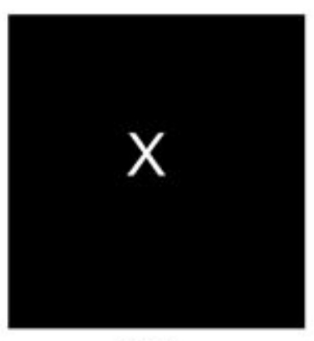

Rest

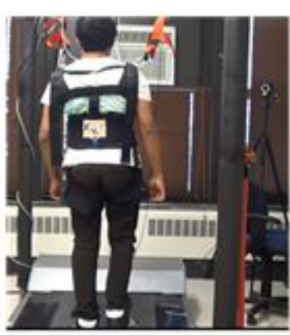

Observed walking

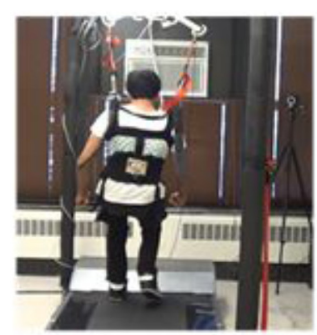

Observed slipping while walking

B

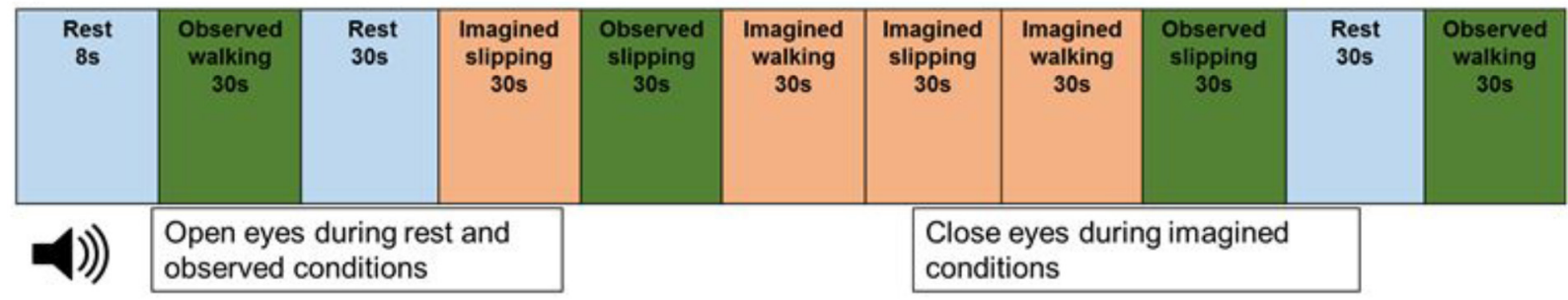

FIGURE 1 | (A) Still images from the video displayed to the subjects while in the scanner, during rest, observed walking and observed slipping while walking. Subject has provided written informed consent for usage of recorded video for data collection during fMRI and images for publication purposes. (B) Order of the rest, observed and imagined conditions used in the fMRI paradigm.

observation from the first person perspective, they were shown videos of back view of walking and slipping, compared with a side view. This was based on the results from a pilot study conducted on a separate set of ten young subjects, which concluded that $90 \%$ of subjects found the back view to be most engaging to view themselves slipping as compared with the side view. The mental imagery of tasks was assessed using the Vividness of Visual Imagery Questionnaire. Following familiarization to the observed and motor imagery tasks, subjects performed these tasks in the MRI scanner. All subjects reported no difficulty in performing mental imagery as indicated by their median score of $4.5 / 5$ on the vividness of visual imagery questionnaire.

\section{Experimental Protocol}

During the MRI scan, subjects were provided with a headset to listen to the instructions and were asked to lie still. Subjects received instructions for the imagined tasks through the headset and a screen in front of them in the scanner projecting the videos during the observed conditions. Four trials for each of the imagined and observed conditions (IW, IS, OW, and OS) were performed in the scanner. The duration of each trial was $30 \mathrm{~s}$. The experimental tasks were interspersed with four trials of "Rest" condition. During the Rest condition, the screen projected the letter " $\mathrm{X}$ " on a black background and subjects were instructed to focus on the letter on the screen, trying not to think about anything else. The Rest condition was included to record the resting brain activity in the absence of observed or imagined tasks. The observed, imagined and rest trials were presented in a randomized order (Figure 1B). All subjects performed the trials in the same randomized order. Two blocks of two trials each were presented for each condition.

\section{Data Acquisition}

Whole brain imaging was performed with a 3.0 T GE Discovery scanner (Milwaukee, WI, United States) using a standard radio frequency coil and $\mathrm{T} 2 *$-weighted pulse sequence. BOLD functional images were collected using a gradient-echo axial echo planar imaging sequence (Glover and Thomason, 2004) at UIC. The following parameters were used: repetition time $=2,000 \mathrm{~m}$, echo time $=22.2 \mathrm{~ms}$, flip angle $=90$ degree, field of view $=22 \mathrm{~cm}$, 64 by 64 matrix, slice thickness $=3 \mathrm{~mm}, 44$ slices. An axial T1 SPGR structural image was obtained for each using 182 axial images $1 \mathrm{~mm}$ in thickness for spatial normalization [minimum TR/TE (9.292 ms/3.77 ms) TI = $450 \mathrm{~ms}$ ]. During scanning, subjects completed the observed and imagined tasks. Prior to scanning, the importance of remaining motionless was conveyed to each subject. There were two runs of the experimental tasks, each lasting $5 \mathrm{~min}$ and $20 \mathrm{~s}$, and acquiring 120 volumes. Each condition was averaged across both runs, prior to the creation of subtraction contrast analyses. The same scanner and acquisition sequence was used for all subjects and there was no relationship between the year the fMRI was performed and extracted activation of the BOLD signal.

\section{Data Processing}

Preprocessing of fMRI data was conducted using SPM8 ${ }^{1}$, FSL, and $\mathrm{AFNI}^{2}$. Data were despiked using AFNI. All data was then slice-time corrected in SPM8 and realigned in $\mathrm{FSL}^{3}$ using MCFLIRT (Jenkinson et al., 2002). Anatomical and functional images were coregistered and normalized to Montreal

\footnotetext{
${ }^{1}$ http://www.fil.ion.ucl.ac.uk/spm/

${ }^{2}$ http://afni.nimh.nih.gov/afni/

${ }^{3}$ http://fsl.fmrib.ox.ac.uk/fsl/fslwiki/
} 
Neurological Institute (MNI) space using SPM8. Smoothing was completed with a full width at half maximum filter of $5 \mathrm{~mm}$. First level models were built in SPM8 using $\mathrm{x}, \mathrm{y}$, and $\mathrm{z}$ translation realignment movement regressors from FSL for each run. The subtraction method was used to create contrast images and second level models were built in SPM8.

\section{Statistical Analysis}

A gray matter mask was applied and whole brain correction was achieved for the multiple regression model at $p=0.01$ by conducting 1000 Monte Carlo simulations in 3dClustSim to determine a joint threshold of height and extent $(p<0.005$, extent of $440 \mathrm{~mm}^{3}$ or $\mathrm{K}=55$ voxels). The Monte Carlo approach was intended to balance Type I and Type II error and cluster extent was determined using the bug-fixed 3dClustSim tool (Cox et al., 2017). Further, the fMRI data was evaluated with planned $t$-test contrasts between selected conditions. Given the above rationales, the $p$-value was not further adjusted for multiple comparisons. We first built contrasts for each active condition by subtracting rest activation from the condition, for example: IW-minus-rest. Then, we compared activation between different conditions using contrasts created relative to the rest condition. For example, we subtracted IW-minus-rest activation from ISminus-rest activation. In total, the following planned contrasts were used: IW-rest, IS-Rest, IS-IW, IW-OW, IS-OS.

\section{RESULTS}

\section{Mental Imagery of Walking and Slipping Versus Rest}

As compared with rest condition (baseline), the cerebral activity was greater in both mental imagery conditions (walking and slipping) (Tables 1, 2). Mental imagery of walking showed increased activity in the frontal lobe within the left supplementary motor area (BA 32). The imagined slipping condition resulted in a significantly greater activity in both cortical and subcortical regions as compared with the rest condition. The cortical areas included left supplementary motor areas (BA6), left superior frontal gyrus, right pars opercularis (BA45), left inferior parietal lobule (BA 40), right parahippocampal gyrus, left cingulate gyrus and right frontal inferior operculum (BA45). Among the sub-cortical structures, bilateral posterior cerebellum showed increased activation during imagined slipping.

\section{Mental Imagery of Slipping Versus Walking}

Imagined slipping resulted in greater activation compared with imagined walking (Table 3 and Figure 2). As compared with imagined walking, imagined slipping showed significantly more activation in left precentral and right middle frontal gyri, bilateral inferior parietal lobule (BA 40), precuneus, bilateral insula, right superior temporal gyrus (BA 22 and 38), right middle and left transverse temporal gyri, right parahippocampal gyrus, bilateral cingulate gyrus, right pulvinar nucleus in thalamus, right cerebellum, and pons in the brainstem.

\section{Mental Imagery Versus Observation of Walking}

The contrast comparing mental imagery of walking to observation of walking did not reveal any active voxels at the specified threshold. Thus, there was no difference in the areas of activation between mental imagery and observation of walking.

\section{Mental Imagery Versus Observation of Slipping}

As compared with observed slipping, the mental imagery of slipping showed additional activation in left supplementary motor area (BA 6), bilateral middle frontal gyrus (BA 10), left superior temporal gyri, bilateral parahippocampal gyrus, left cingulate gyrus. (Table 4 and Figure 3). Also, as opposed to observation of slipping, mental imagery of slipping yielded greater activation in the left inferior parietal lobule. Both mental imagery and observation of slipping showed similar activation in the inferior frontal gyrus and limbic regions.

\section{DISCUSSION}

The purpose of this pilot study was to examine the neural correlates associated with slipping while walking. As hypothesized, activation of additional brain areas was seen during imagined slipping as compared with imagined walking. Our other major findings include increased activation during imagined slipping as compared with observed slipping and no additional activation in imagined walking as compared to observed walking.

\section{Mental Imagery of Slipping Versus Walking}

The middle frontal gyrus was the common area activated between the imagined walk minus rest and imagined slip minus rest contrasts. However, imagined slip condition showed additional activation in areas such as supplementary motor area (SMA), dorsolateral prefrontal cortex, inferior parietal lobule, cerebellum, precuneus, cingulate gyrus, parahippocampal gyri and pulvinar nucleus of the thalamus over imagined walking. The brain activation pattern noted for imagined slipping was remarkably similar to previous studies which found activation in SMA, frontal cortex and cerebellum during imagery of dynamic balancing tasks (Ferraye et al., 2014; Taube et al., 2015).

The increased activation in the SMA during imagined slip condition over the imagined walk condition could be assumed as a response to perturbation causing activation of the SMA-cerebellar loop via the pontine nucleus and back to the motor cortex or association area in order to modify the ongoing movement. SMA, is mainly related to movement execution, motor planning and coordination along with dorsolateral prefrontal cortex, which help in response selection using prior associations (Eccles, 1982; Chouinard and Paus, 2006). These areas generate an appropriate motor response, including corrective motor responses, to prevent loss 
TABLE 1 | Differences in activation between imagined slipping and rest conditions.

\begin{tabular}{|c|c|c|c|c|c|c|c|c|c|c|}
\hline \multirow[t]{2}{*}{ Contrast } & \multicolumn{4}{|c|}{ Anatomical Regions } & \multirow[t]{2}{*}{$\mathrm{mm}^{3}$} & \multirow[t]{2}{*}{ Voxels } & \multicolumn{3}{|c|}{ MNI coordinates } & \multirow[b]{2}{*}{ Z-value } \\
\hline & Lobe & Gyrus & BA & Side & & & $x$ & $y$ & $z$ & \\
\hline \multirow[t]{8}{*}{ IS > Rest } & Frontal & $\begin{array}{l}\text { Precentral Frontal } \\
\text { inferior operculum }\end{array}$ & - & $\mathrm{L}$ & 6,112 & 764 & -48 & 8 & 14 & 3.87 \\
\hline & & Superior frontal & 6 & $L$ & 1,040 & 130 & -32 & 12 & 54 & 3.80 \\
\hline & Parietal & $\begin{array}{l}\text { Frontal inferior } \\
\text { operculum }\end{array}$ & 45 & $\mathrm{R}$ & 2,608 & 326 & 56 & 10 & 22 & 3.92 \\
\hline & & Inferior parietal lobule & 40 & $\mathrm{~L}$ & 920 & 115 & -46 & -46 & 46 & 3.48 \\
\hline & Limbic & Parahippocampal & - & $\mathrm{R}$ & 640 & 80 & 14 & -10 & -18 & 3.34 \\
\hline & & Cingulate gyrus & 24 & $L$ & 10,352 & 1294 & -4 & -8 & 50 & 4.07 \\
\hline & Cerebellum - & Declive Cerebellum and & $\mathrm{N} / \mathrm{A}$ & $L$ & 904 & 113 & -38 & -60 & -30 & 3.54 \\
\hline & posterior lobe & crus cerebri & & $\mathrm{R}$ & 1,016 & 127 & 28 & -74 & -28 & 3.77 \\
\hline
\end{tabular}

IS, Imagined slipping; BA, Broadmann area.

TABLE 2 | Differences in activation between imagined walking and rest conditions.

\begin{tabular}{|c|c|c|c|c|c|c|c|c|c|c|}
\hline \multirow[t]{2}{*}{ Contrast } & \multicolumn{4}{|c|}{ Anatomical regions } & \multirow[t]{2}{*}{$\mathrm{mm}^{3}$} & \multirow[t]{2}{*}{ Voxels } & \multicolumn{3}{|c|}{ MNI coordinates } & \multirow[b]{2}{*}{ Z-value } \\
\hline & Lobe & Gyrus & BA & Side & & & $x$ & $y$ & $z$ & \\
\hline IW > Rest & Frontal & Middle frontal & 32 & $\mathrm{~L}$ & 808 & 101 & -6 & 6 & 50 & 3.49 \\
\hline
\end{tabular}

IW, Imagined walking; BA, Broadmann area.

TABLE 3 | Differences in activation between imagined slipping and imagined walking.

\begin{tabular}{|c|c|c|c|c|c|c|c|c|c|c|}
\hline \multirow[t]{2}{*}{ Contrast } & \multicolumn{4}{|c|}{ Anatomical regions } & \multirow[t]{2}{*}{$\mathrm{mm}^{3}$} & \multirow[t]{2}{*}{ Voxels } & \multicolumn{3}{|c|}{ MNI coordinates } & \multirow[b]{2}{*}{$Z$-value } \\
\hline & Lobe & Gyrus & BA & Side & & & $x$ & $y$ & $z$ & \\
\hline \multirow[t]{19}{*}{$\mathrm{IS}>\mathrm{IW}$} & \multirow[t]{2}{*}{ Frontal } & Precentral & - & $L$ & 704 & 88 & -52 & -4 & 6 & 3.38 \\
\hline & & Middle frontal & - & $\mathrm{R}$ & 968 & 121 & 42 & -4 & 48 & 3.33 \\
\hline & \multirow[t]{3}{*}{ Parietal } & Inferior parietal & 40 & $\mathrm{R}$ & 1,288 & 161 & 56 & -34 & 24 & 3.32 \\
\hline & & lobule & 40 & $L$ & 704 & 88 & -56 & -30 & 24 & 3.41 \\
\hline & & Precuneus & 7 & & 480 & 60 & -8 & -64 & 56 & 3.15 \\
\hline & \multirow[t]{4}{*}{ Temporal } & Superior temporal & 38 & $\mathrm{R}$ & 440 & 55 & 36 & 6 & -22 & 3.84 \\
\hline & & & 22 & $\mathrm{R}$ & 720 & 90 & 46 & -18 & 0 & 3.22 \\
\hline & & Middle temporal & - & $\mathrm{R}$ & 848 & 106 & 54 & -42 & 0 & 3.79 \\
\hline & & $\begin{array}{l}\text { Transverse } \\
\text { temporal }\end{array}$ & - & $\mathrm{L}$ & 1,080 & 135 & -38 & -28 & 12 & 4.17 \\
\hline & \multirow[t]{3}{*}{ Limbic } & Parahippocampal & 35 & $\mathrm{R}$ & 1,056 & 132 & 18 & -30 & -10 & 4.00 \\
\hline & & Cingulate & 31 & $\mathrm{R}$ & 984 & 123 & 8 & -30 & 42 & 3.19 \\
\hline & & & & $L$ & 768 & 96 & -6 & -34 & 42 & 3.25 \\
\hline & \multirow[t]{3}{*}{ Insula } & \multirow{3}{*}{$\begin{array}{l}\text { Insula L Rolandic } \\
\text { Oper Undefined }\end{array}$} & \multirow[t]{3}{*}{-} & $\mathrm{L}$ & 624 & 78 & -40 & 0 & 0 & 3.35 \\
\hline & & & & $L$ & 1,048 & 131 & -44 & 4 & 14 & 4.16 \\
\hline & & & & $\mathrm{R}$ & 928 & 116 & 34 & 10 & 10 & 3.62 \\
\hline & Thalamus & Pulvinar & - & $\mathrm{R}$ & 640 & 80 & 8 & -28 & 2 & 4.01 \\
\hline & Brainstem & Pons & - & $L$ & 1,256 & 157 & -8 & -32 & -24 & 3.85 \\
\hline & Cerebellum Anterior & Undefined Declive & - & $\mathrm{R}$ & 600 & 75 & 18 & -42 & -30 & 3.30 \\
\hline & $\begin{array}{l}\text { Lobe Posterior } \\
\text { Lobe }\end{array}$ & of vermis & NA & $\mathrm{R}$ & 984 & 123 & 2 & -70 & -26 & 3.14 \\
\hline
\end{tabular}

IS, imagined slipping; IW, imagined walking; BA, Broadmann area.

of balance or fall. Our findings are in line with EEG studies performed during standing perturbations, which indicated the presence of N1, negative event-related potential occurring about $100 \mathrm{~ms}$ after onset of an unpredictable stance perturbation in the fronto-central cortical sites (Quant et al., 2004; Adkin et al., 2006; Mochizuki et al., 2009). In these studies, small magnitude perturbations were delivered which evoked feet-inplace postural responses. In addition, it was found that the 


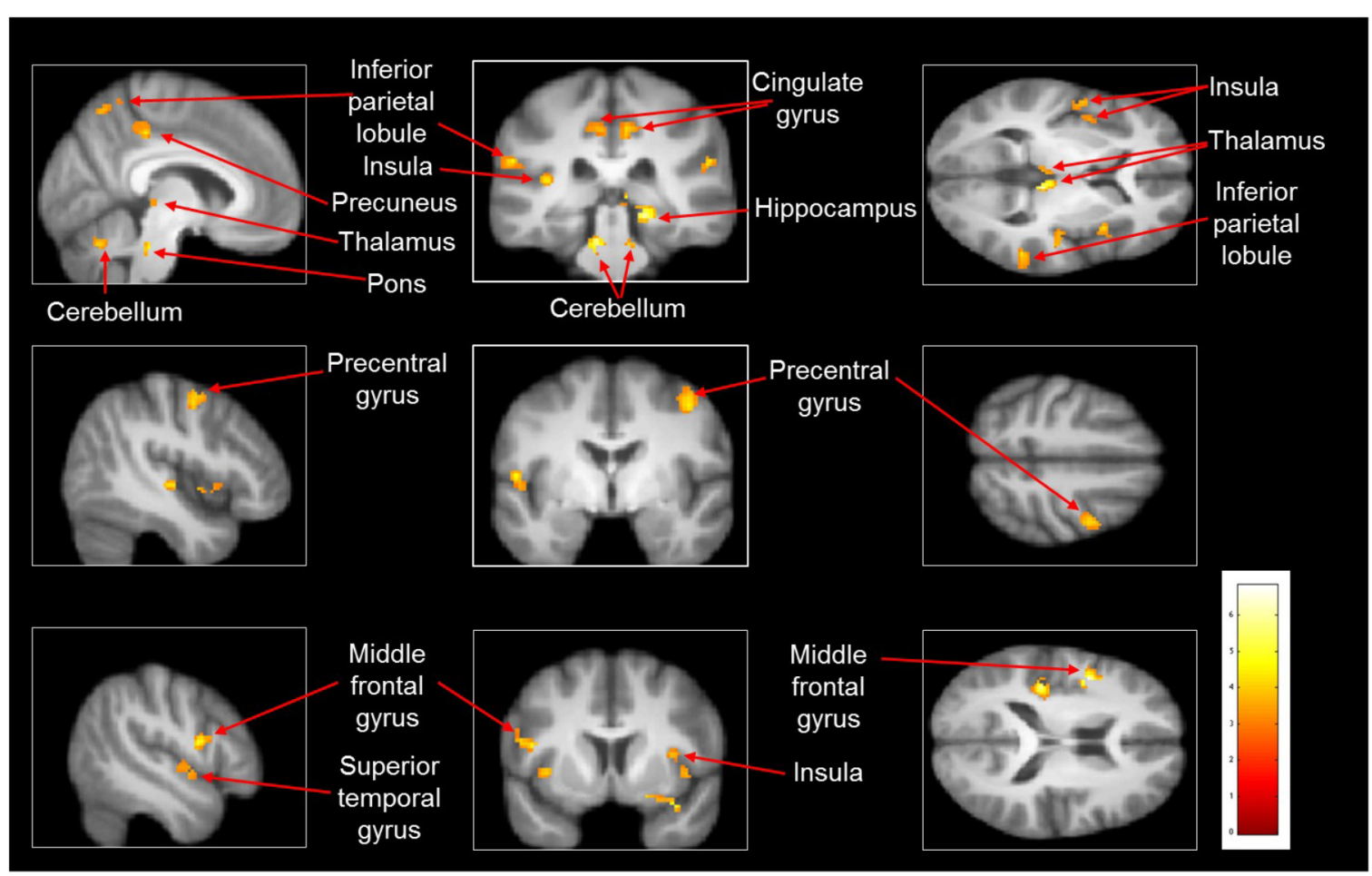

FIGURE 2 | Heat map demonstrating areas with greater activation in imagined slipping versus imagined walking. Increased activation was noted in the precentral and middle frontal gyri, inferior parietal lobule (BA 40), precuneus, superior temporal gyrus (BA 22 and 38), parahippocampal gyrus, cingulate gyrus, pulvinar nucleus in thalamus, bilateral cerebellum, and pons in brainstem $(p<0.005)$.

TABLE 4 | Differences in activation between imagined slipping and observed slipping.

\begin{tabular}{|c|c|c|c|c|c|c|c|c|c|c|}
\hline \multirow[t]{2}{*}{ Contrast } & \multicolumn{4}{|c|}{ Anatomical regions } & \multirow[t]{2}{*}{$\mathrm{mm}^{3}$} & \multirow[t]{2}{*}{ Voxels } & \multicolumn{3}{|c|}{ MNI coordinates } & \multirow[b]{2}{*}{$Z$-value } \\
\hline & Lobe & Gyrus & BA & Side & & & $x$ & $y$ & $z$ & \\
\hline \multirow[t]{10}{*}{ IS $>$ OS } & Frontal & Middle frontal & - & $\mathrm{R}$ & 2,768 & 346 & 36 & 32 & 26 & 4.28 \\
\hline & & & 10 & $\mathrm{~L}$ & 1,368 & 171 & -36 & 40 & 24 & 3.46 \\
\hline & & Inferior frontal & - & $L$ & 4,280 & 535 & -36 & 30 & 2 & 3.85 \\
\hline & Parietal & $\begin{array}{l}\text { Inferior Parietal } \\
\text { lobule Extra } \\
\text { Nuclear }\end{array}$ & 40 & $R$ & 536 & 67 & 64 & -42 & 32 & 4.10 \\
\hline & & & - & $L$ & 624 & 78 & -16 & 2 & 14 & 3.70 \\
\hline & Temporal & Superior temporal & & $L$ & 9,584 & 1198 & -52 & -18 & 0 & 4.17 \\
\hline & Limbic & Parahippocampal & - & $R$ & 13,160 & 1645 & 36 & -16 & -28 & 4.46 \\
\hline & & & & $L$ & 1,720 & 215 & -16 & -36 & -12 & 3.95 \\
\hline & & & & $\mathrm{R}$ & 1,328 & 166 & 20 & -20 & -16 & 3.63 \\
\hline & & Cingulate (SMA) & - & $L$ & 27,216 & 3402 & -4 & 4 & 46 & 5.09 \\
\hline
\end{tabular}

IS: Imagined slipping, OS: Observed slipping, BA: Broadmann area, SMA: Supplementary motor areas.

amplitude of the N1 response changed with the knowledge of an impending perturbation such that the N1 amplitude was greater during an unpredictable perturbation (Adkin et al., 2006). Similarly, Mihara et al. (2012) using fNIRS, examined cortical activation during anterior-posterior and medio-lateral stance perturbations in individuals with stroke. It was found that postural responses were accompanied by increased uptake of oxygenated hemoglobin in bilateral prefrontal, premotor and parietal cortex association areas. Thus, these studies suggest the role of frontal cortex in detection of balance loss and in modulating recovery responses (Dietz et al., 1984).

In order to correct movement error for restoring and maintaining the COM within the BOS, the cerebellum could be activated. Since cerebellum (vermis) acts as a comparator for signals received from the proprioceptors of the moving 


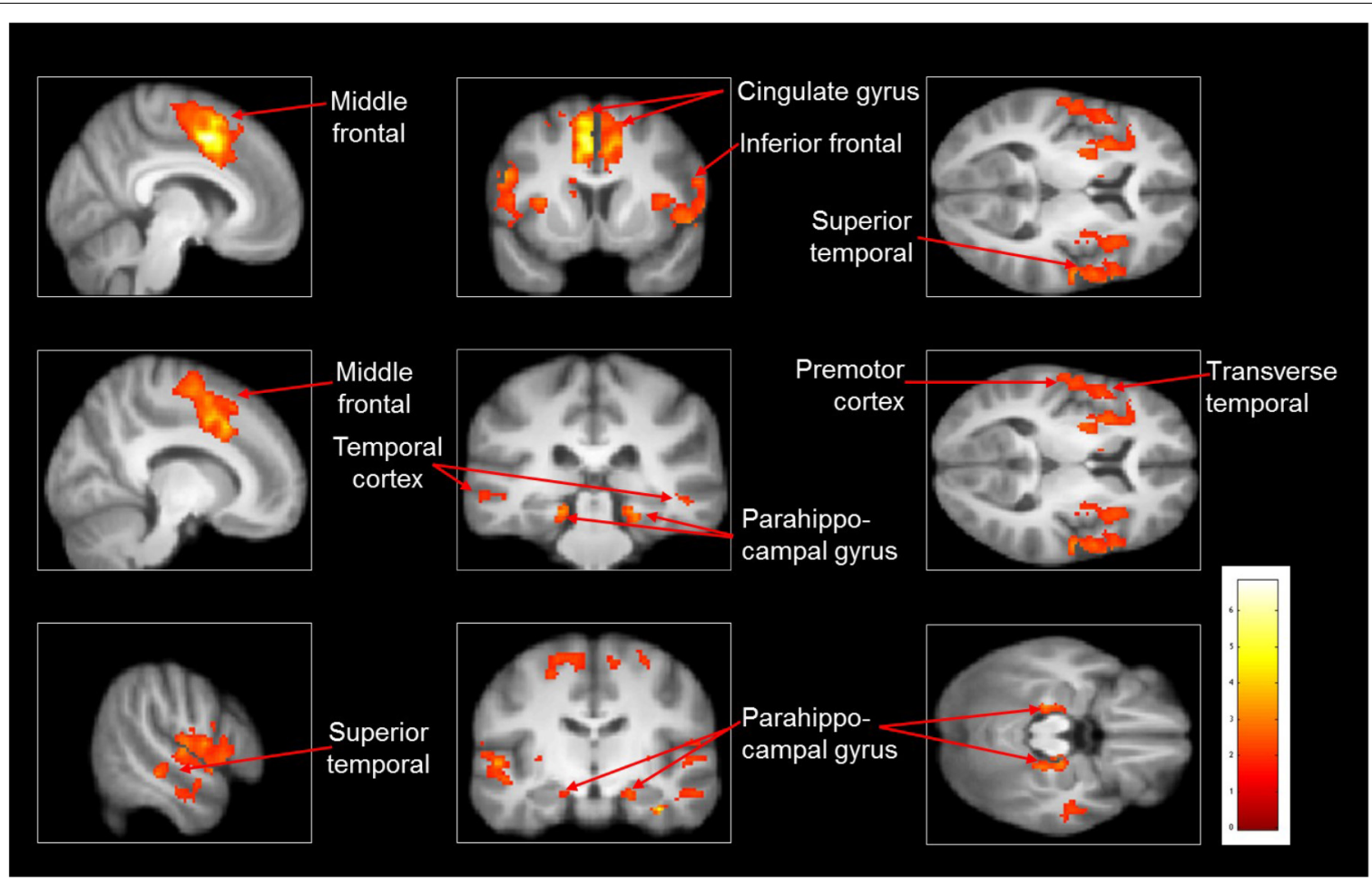

FIGURE 3 | Heat map showing areas with increased activation in the imagined slipping compared with observed slipping. Additional activation was noted in supplementary motor area (BA 6), anterior prefrontal cortex (BA 10), superior temporal gyri, bilateral parahippocampal gyrus, cingulate gyrus, and inferior parietal lobule $(p<0.005)$ in imagined slipping versus observed slipping.

body segments via the spinocerebellar tract and timely modulate the response to match the intended movement, it could be involved in planning of the reactive stepping response (Pisotta and Molinari, 2014).

We found activation of areas related to visuo-spatial navigation such as the precuneus region of the parietal lobe and parahippocampal gyri. Activation in these regions could be related to the mental visualization of the slipping environment (Cavanna and Trimble, 2006) and formation of associations between visual and spatial information, thus helping in integration of the walking path (McNaughton et al., 1996). Furthermore, parahippocampal gyri could also be associated to the retrieval of past memory in order to generate the reactive balance responses (Ekstrom and Bookheimer, 2007).

Considering the unique sensory experience provided by slipping, the activation of inferior parietal lobule could be associated with the integration of somatosensory and visual stimuli (Singh-Curry and Husain, 2009). Similarly, activation of thalamus could be attributed to sensory integration, processing and maintaining erect posture while recovering balance from falls (Zwergal et al., 2009; Barra et al., 2010). Activation in insula which helps in perception, motor control and self-awareness was also noted (Sterzer and Kleinschmidt, 2010).

There was significant activation in the cingulate gyrus, which plays a role in monitoring error while performing motor tasks and could be of significance in detecting balance loss during walking (Holroyd et al., 2004; Debener et al., 2005; Anguera et al., 2009; Sipp et al., 2013). Our findings are similar to the results from a recent study, which used high-density EEG along with independent component analysis to identify cortical activity related to loss of balance (Sipp et al., 2013). The authors found that walking on a balance beam mounted on a treadmill was associated with activation of anterior cingulate, posterior cingulate, anterior parietal, sensorimotor and dorsolateral-prefrontal cortices (Sipp et al., 2013).

Despite some similarities in activations observed between imagined slipping and walking conditions, additional cortical and subcortical areas recruitment during imagined slipping suggest the involvement of different neural pathways in reactive balance control while walking than regular walking. This could be functionally or in real-life associated with the novelty of the sensorimotor stimulus and the perceived complexity of maintaining stability during a sudden slip while walking. It is established that longer-term training of a motor skill reduces the amount of cortical activation in motor areas suggesting a more efficient control of movement after sufficient practice (Jancke et al., 2000; Picard et al., 2013; Naito and Hirose, 2014). On similar lines, walking is a self-generated, anticipatory and a relatively learned (over years) task as compared with recovering balance during a sudden slip. Unlike regular walking, an unexpected disturbance during walking results in larger excursion of COM threatening 
postural stability. Such disturbance while walking might necessitate recruitment of a variable motor command than regular walking to control lower extremities and execute an appropriate postural response. It is thus likely that, slipping demands greater neural resources for sensory (visuospatial, perceptual, tactile, proprioceptive), cognitive and motor processing than regular walking, as that observed in our study.

\section{Mental Imagery Versus Observation of Walking}

Neuroimaging studies have established the existence of a strong overlap in the neural areas activated during the actual task performance and its imagination and observation (Buccino et al., 2001; Grezes and Decety, 2002; Hanakawa et al., 2003). This evidence comes from studies examining brain activation during lower extremity movements (la Fougere et al., 2010; Dalla Volta et al., 2015). For example, Iseki et al. (2008) compared imagined and observed walking, and found that SMA and the dorsal premotor cortex (PMd) were activated in both the conditions (Iseki et al., 2008). Previous studies have shown that these two areas are essential for gait initiation, turning, negotiating obstacles and walking in narrow passages (Della Sala et al., 2002; Malouin et al., 2003; Nadeau, 2007). Another study examining neural activation during whole body gymnastic movements showed that similar motor areas were activated during both motor imagery and action observation (Munzert et al., 2008). Furthermore, Orr et al. (2008) observed that both imagination and observation of ankle movements was associated with common activation in inferior parietal lobule and precentral gyrus. Based on previous evidence, the fact that we did not observe any additional activation in imagined versus observed walking condition could be due to the common areas activated during these conditions.

\section{Mental Imagery Versus Observation of Slipping}

Contrary to that seen for regular walking, for the slipping condition, our results suggest that there was additional activation noted during imagined versus the observed condition. Although motor imagery and observation could be driven by similar neural pathways (Grafton et al., 1996; Grezes and Decety, 2001; Jeannerod, 2001), it can be argued that first person motor imagery involves a more conscious brain engagement in an attempt to simulate the actual motor experience. This may be particularly important during tasks posing significant threat to balance and the CNS relies on activation of necessary visualperceptual and motor systems to select an appropriate response. In our study, imagined slipping showed additional activation in SMA (BA 6) and bilateral middle frontal gyrus, which are involved in movement planning and initiation (Eccles, 1982; Chouinard and Paus, 2006); cingulate gyrus, which monitors motor error and detects balance loss during walking (Holroyd et al., 2004; Debener et al., 2005; Anguera et al., 2009; Sipp et al., 2013) and inferior frontal gyrus, which is involved in regulating excessive trunk movement to prevent loss of balance via response suppression (Sharp et al., 2010). Furthermore, there was recruitment of sensory and visual processing areas such as superior temporal gyri, bilateral parahippocampal gyrus and cingulate gyrus, (McNaughton et al., 1996; Yang et al., 2015) and areas related to perception and internal awareness such as inferior parietal lobule, resulting in increased activation (SinghCurry and Husain, 2009). These findings clearly suggest greater engagement of neural structures while imagining a challenging locomotor-balance task like slipping. Our findings are also in agreement with the study by Taube et al. (2015) showing increased activation in SMA and the middle frontal gyrus during imagery over observation of maintaining standing balance on a wobble board which continuously disturbs balance. Thus, current and previous findings highlight that the extent of neural activation during motor imagery might also be influenced by task complexity, uniqueness or prior motor experience with the task.

\section{CLINICAL APPLICATION AND LIMITATION}

Considering that mental imagery showed greater activation of brain areas, it can be used to better examine the neural correlates than action observation for activities which cannot be overtly performed in the scanner. While a mental imagery paradigm is beneficial to evaluate gait related activities, the findings of this study should be interpreted in light of few limitations of this approach. There could be individual differences in the ability of the subjects to imagine even though no difference was noted on the scoring of the Vividness of motor and visual imagery questionnaire, which can alter the areas and the extent of clusters activated, as supported by van der Meulen et al. (2014). Furthermore, the quality of the imagined movement is dependent on the attentiveness during imagination (De Beni et al., 2007; Gregg et al., 2010). The elimination of action of gravity on the otherwise weight bearing activities of walking and slipping may have some effect on the overall activation pattern. Also, the observed differences between activations during mental imagery and rest or observed conditions could be due to the fact that the eyes were closed in the imagined condition and open during rest and observed conditions.

Since our study is a pilot study, the study may be underpowered owing to a smaller sample size. However, this pilot study does provide a proof of feasibility, and estimates of effect sizes, so that larger studies, needed to rule out type I error can be planned. To evaluate potential future clinical application of our results in other population, a larger sample size with variable hand dominance would be recommended. Further, examining those with known gait disturbances can also provide convergent validity for these results. In spite of these limitations, we had the advantage of better spatial resolution as compared with other imaging techniques provided by use of $f M R I$ (Chen and Ugurbil, 1999). 
The results of this study suggest that a network of cortical and subcortical areas somewhat different from regular walking is associated with the act of balancing in response to a novel slip. It further corroborates the view that specific cortical regions play a role in reactive balance control which has been proposed by several behavioral (Rankin et al., 2000; Brauer et al., 2002; Adkin et al., 2006) and animal studies (Beloozerova and Sirota, 1993; Deliagina et al., 2007). This study elucidates the neural structures associated with slipping in a young healthy nervous system. Future studies should investigate the brain activation patterns in elderly and in populations with balance deficits to further inform neural mechanisms of reactive balance control and potential causes of falls in these individuals.

\section{DISCLOSURE}

No commercial party having a direct financial interest in the research findings reported here has conferred or will confer a benefit on the authors or on any organization with which the authors are associated.

\section{REFERENCES}

Adkin, A. L., Quant, S., Maki, B. E., and McIlroy, W. E. (2006). Cortical responses associated with predictable and unpredictable compensatory balance reactions. Exp. Brain Res. 172, 85-93. doi: 10.1007/s00221-005-0310-9

Allum, J., and Honegger, F. (1992). A postural model of balance-correcting movement strategies. J. Vestib. Res. 2, 323-347.

Anguera, J. A., Seidler, R. D., and Gehring, W. J. (2009). Changes in performance monitoring during sensorimotor adaptation. J. Neurophysiol. 102, 1868-1879. doi: 10.1152/jn.00063.2009

Armstrong, D. M., and Drew, T. (1985). Forelimb electromyographic responses to motor cortex stimulation during locomotion in the cat. J. Physiol. 367, 327-351. doi: 10.1113/jphysiol.1985.sp015827

Aruin, A. S., and Latash, M. L. (1995). The role of motor action in anticipatory postural adjustments studied with self-induced and externally triggered perturbations. Exp. Brain Res. 106, 291-300. doi: 10.1007/BF00241125

Barra, J., Marquer, A., Joassin, R., Reymond, C., Metge, L., Chauvineau, V., et al. (2010). Humans use internal models to construct and update a sense of verticality. Brain 133(Pt 12), 3552-3563. doi: 10.1093/brain/awq311

Beloozerova, I. N., and Sirota, M. G. (1993). The role of the motor cortex in the control of accuracy of locomotor movements in the cat. J. Physiol. 461, 1-25. doi: 10.1113/jphysiol.1993.sp019498

Berger, W., Dietz, V., and Quintern, J. (1984). Corrective reactions to stumbling in man: neuronal co-ordination of bilateral leg muscle activity during gait. J. Physiol. 357, 109-125. doi: 10.1113/jphysiol.1984.sp015492

Bhatt, T., and Pai, Y. C. (2008). Can observational training substitute motor training in preventing backward balance loss after an unexpected slip during walking? J. Neurophysiol. 99, 843-852. doi: 10.1152/jn.00720. 2007

Brauer, S. G., Woollacott, M., and Shumway-Cook, A. (2002). The influence of a concurrent cognitive task on the compensatory stepping response to a perturbation in balance-impaired and healthy elders. Gait Posture 15, 83-93. doi: 10.1016/S0966-6362(01)00163-1

Buccino, G. (2014). Action observation treatment: a novel tool in neurorehabilitation. Philos. Trans. R. Soc. Lond. B Biol. Sci. 369:20130185. doi: 10.1098/rstb.2013.0185

Buccino, G., Binkofski, F., Fink, G. R., Fadiga, L., Fogassi, L., Gallese, V., et al. (2001). Action observation activates premotor and parietal areas in a somatotopic manner: an fMRI study. Eur. J. Neurosci. 13, 400-404. doi: 10.1046/ j.1460-9568.2001.01385.x

\section{ETHICS STATEMENT}

A written informed consent was obtained from all research participants. The study protocol was approved by the Institutional Review at University of Illinois at Chicago.

\section{AUTHOR CONTRIBUTIONS}

TB provided the conceptual framework and experimental design, facilities, funding, and assistance with manuscript preparation. SL has contributed toward experimental design, data processing and analysis, consultation and reviewing of manuscript. PP and SD assisted with experimental design, data collection, analysis, and manuscript preparation. SRD has assisted with data processing, analysis, and manuscript review.

\section{ACKNOWLEDGMENTS}

We would like to thank Mike Flannery for his guidance and assistance with MRI data collection.

Caligiore, D., Mustile, M., Spalletta, G., and Baldassarre, G. (2017). Action observation and motor imagery for rehabilitation in Parkinson's disease: a systematic review and an integrative hypothesis. Neurosci. Biobehav. Rev. 72, 210-222. doi: 10.1016/j.neubiorev.2016.11.005

Cavanna, A. E., and Trimble, M. R. (2006). The precuneus: a review of its functional anatomy and behavioural correlates. Brain 129(Pt 3), 564-583. doi: 10.1093/ brain/awl004

Chen, W., and Ugurbil, K. (1999). High spatial resolution functional magnetic resonance imaging at very-high-magnetic field. Top. Magn. Reson. Imaging 10, 63-78. doi: 10.1097/00002142-199902000-00006

Chouinard, P. A., and Paus, T. (2006). The primary motor and premotor areas of the human cerebral cortex. Neuroscientist 12, 143-152. doi: 10.1177/ 1073858405284255

Cox, R. W., Chen, G., Glen, D. R., Reynolds, R. C., and Taylor, P. A. (2017). FMRI clustering in AFNI: false-positive rates redux. Brain Connect. 7, 152-171. doi: 10.1089/brain.2016.0475

Dalla Volta, R., Fasano, F., Cerasa, A., Mangone, G., Quattrone, A., and Buccino, G. (2015). Walking indoors, walking outdoors: an fMRI study. Front. Psychol. 6:1502. doi: 10.3389/fpsyg.2015.01502

De Beni, R., Pazzaglia, F., and Gardini, S. (2007). The generation and maintenance of visual mental images: evidence from image type and aging. Brain Cogn. 63, 271-278. doi: 10.1016/j.bandc.2006.09.004

Debener, S., Ullsperger, M., Siegel, M., Fiehler, K., von Cramon, D. Y., and Engel, A. K. (2005). Trial-by-trial coupling of concurrent electroencephalogram and functional magnetic resonance imaging identifies the dynamics of performance monitoring. J. Neurosci. 25, 11730-11737. doi: 10.1523/jneurosci.3286-05.2005

Decety, J., and Jeannerod, M. (1995). [Imagery and its neurological substrate]. Rev. Neurol. 151, 474-479.

Deliagina, T. G., Zelenin, P. V., Beloozerova, I. N., and Orlovsky, G. N. (2007). Nervous mechanisms controlling body posture. Physiol. Behav. 92, 148-154. doi: 10.1016/j.physbeh.2007.05.023

Della Sala, S., Francescani, A., and Spinnler, H. (2002). Gait apraxia after bilateral supplementary motor area lesion. J. Neurol. Neurosurg. Psychiatry 72, 77-85. doi: 10.1136/jnnp.72.1.77

Diener, H. C., Horak, F. B., and Nashner, L. M. (1988). Influence of stimulus parameters on human postural responses. J. Neurophysiol. 59, 1888-1905. doi: 10.1152/jn.1988.59.6.1888

Dietz, V., Quintern, J., and Berger, W. (1984). Cerebral evoked potentials associated with the compensatory reactions following stance and gait perturbation. Neurosci. Lett. 50, 181-186. doi: 10.1016/0304-3940(84)90483-X 
Dietz, V., Quintern, J., Boos, G., and Berger, W. (1986). Obstruction of the swing phase during gait: phase-dependent bilateral leg muscle coordination. Brain Res. 384, 166-169. doi: 10.1016/0006-8993(86)91233-3

Drew, T. (1988). Motor cortical cell discharge during voluntary gait modification. Brain Res. 457, 181-187. doi: 10.1016/0006-8993(88)90073-X

Eccles, J. C. (1982). The initiation of voluntary movements by the supplementary motor area. Arch. Psychiatr. Nervenkr. 231, 423-441. doi: 10.1007/BF00342722

Edwards, M. G., Humphreys, G. W., and Castiello, U. (2003). Motor facilitation following action observation: a behavioural study in prehensile action. Brain Cogn. 53, 495-502. doi: 10.1016/S0278-2626(03)00210-0

Ekstrom, A. D., and Bookheimer, S. Y. (2007). Spatial and temporal episodic memory retrieval recruit dissociable functional networks in the human brain. Learn. Mem. 14, 645-654. doi: 10.1101/lm.575107

Eng, J. J., Winter, D. A., and Patla, A. E. (1994). Strategies for recovery from a trip in early and late swing during human walking. Exp. Brain Res. 102, 339-349. doi: 10.1007/BF00227520

Ferber, R., Osternig, L. R., Woollacott, M. H., Wasielewski, N. J., and Lee, J.H. (2002). Reactive balance adjustments to unexpected perturbations during human walking. Gait Posture 16, 238-248. doi: 10.1016/S0966-6362(02)000103

Ferraye, M. U., Debu, B., Heil, L., Carpenter, M., Bloem, B. R., and Toni, I. (2014). Using motor imagery to study the neural substrates of dynamic balance. PLoS One 9:e91183. doi: 10.1371/journal.pone.0091183

Forssberg, H., Grillner, S., and Rossignol, S. (1975). Phase dependent reflex reversal during walking in chronic spinal cats. Brain Res. 85, 103-107. doi: 10.1016/ 0006-8993(75)91013-6

Franceschini, M., Agosti, M., Cantagallo, A., Sale, P., Mancuso, M., and Buccino, G. (2010). Mirror neurons: action observation treatment as a tool in stroke rehabilitation. Eur. J. Phys. Rehab. Med. 46, 517-523.

Garcia Carrasco, D., and Aboitiz Cantalapiedra, J. (2016). Effectiveness of motor imagery or mental practice in functional recovery after stroke: a systematic review. Neurologia 31, 43-52. doi: 10.1016/j.nrl.2013.02.003

Glover, G. H., and Thomason, M. E. (2004). Improved combination of spiral-in/out images for BOLD fMRI. Magn. Reson. Med. 51, 863-868. doi: 10.1002/mrm. 20016

Grafton, S. T., Arbib, M. A., Fadiga, L., and Rizzolatti, G. (1996). Localization of grasp representations in humans by positron emission tomography. 2 . Observation compared with imagination. Exp. Brain Res. 112, 103-111. doi: 10.1007/BF00227183

Graydon, F. X., Friston, K. J., Thomas, C. G., Brooks, V. B., and Menon, R. S. (2005). Learning-related fMRI activation associated with a rotational visuomotor transformation. Brain Res. Cogn. Brain Res. 22, 373-383. doi: 10.1016/ j.cogbrainres.2004.09.007

Gregg, M., Hall, C., and Butler, A. (2010). The MIQ-RS: a suitable option for examining movement imagery ability. Evid. Based Complement. Alternat. Med. 7, 249-257. doi: 10.1093/ecam/nem170

Grezes, J., and Decety, J. (2001). Functional anatomy of execution, mental simulation, observation, and verb generation of actions: a meta-analysis. Hum. Brain Mapp. 12, 1-19. doi: 10.1002/1097-0193(200101)12:1<1::AID-HBM10> 3.0.CO;2-V

Grezes, J., and Decety, J. (2002). Does visual perception of object afford action? Evidence from a neuroimaging study. Neuropsychologia 40, 212-222. doi: 10. 1016/S0028-3932(01)00089-6

Hanakawa, T., Immisch, I., Toma, K., Dimyan, M. A., Van Gelderen, P., and Hallett, M. (2003). Functional properties of brain areas associated with motor execution and imagery. J. Neurophysiol. 89, 989-1002. doi: 10.1152/jn.00132. 2002

Hardwick, R. M., and Edwards, M. G. (2011). Observed reach trajectory influences executed reach kinematics in prehension. Q. J. Exp. Psychol. 64, 1082-1093. doi: 10.1080/17470218.2010.538068

Holroyd, C. B., Nieuwenhuis, S., Yeung, N., Nystrom, L., Mars, R. B., Coles, M. G., et al. (2004). Dorsal anterior cingulate cortex shows fMRI response to internal and external error signals. Nat. Neurosci. 7, 497-498. doi: 10.1038/ nn 1238

Hong, I. K., Choi, J. B., and Lee, J. H. (2012). Cortical changes after mental imagery training combined with electromyography-triggered electrical stimulation in patients with chronic stroke. Stroke 43, 2506-2509. doi: 10.1161/strokeaha.112. 663641
Horak, F. B. (2006). Postural orientation and equilibrium: what do we need to know about neural control of balance to prevent falls? Age Ageing 35(Suppl. 2), ii7-ii11. doi: 10.1093/ageing/afl077

Ietswaart, M., Johnston, M., Dijkerman, H. C., Joice, S., Scott, C. L., MacWalter, R. S., et al. (2011). Mental practice with motor imagery in stroke recovery: randomized controlled trial of efficacy. Brain 134(Pt 5), 1373-1386. doi: 10. 1093/brain/awr077

Iseki, K., Hanakawa, T., Shinozaki, J., Nankaku, M., and Fukuyama, H. (2008). Neural mechanisms involved in mental imagery and observation of gait. Neuroimage 41, 1021-1031. doi: 10.1016/j.neuroimage.2008.03.010

Jacobs, J. V., and Horak, F. B. (2007). Cortical control of postural responses. J. Neural Transm. 114, 1339-1348. doi: 10.1007/s00702-007-0657-0

Jahn, K., Deutschlander, A., Stephan, T., Kalla, R., Wiesmann, M., Strupp, M., et al. (2008). Imaging human supraspinal locomotor centers in brainstem and cerebellum. Neuroimage 39, 786-792. doi: 10.1016/j.neuroimage.2007.09.047

Jahn, K., Deutschlander, A., Stephan, T., Strupp, M., Wiesmann, M., and Brandt, T. (2004). Brain activation patterns during imagined stance and locomotion in functional magnetic resonance imaging. Neuroimage 22, 1722-1731. doi: 10. 1016/j.neuroimage.2004.05.017

Jancke, L., Shah, N. J., and Peters, M. (2000). Cortical activations in primary and secondary motor areas for complex bimanual movements in professional pianists. Brain Res. Cogn. Brain Res. 10, 177-183. doi: 10.1016/S0926-6410(00) 00028-8

Jeannerod, M. (2001). Neural simulation of action: a unifying mechanism for motor cognition. Neuroimage 14(1 Pt 2), S103-S109. doi: 10.1006/nimg.2001. 0832

Jenkinson, M., Bannister, P., Brady, M., and Smith, S. (2002). Improved optimization for the robust and accurate linear registration and motion correction of brain images. Neuroimage 17, 825-841. doi: 10.1006/nimg.2002. 1132

la Fougere, C., Zwergal, A., Rominger, A., Forster, S., Fesl, G., Dieterich, M., et al. (2010). Real versus imagined locomotion: a [18F]-FDG PET-fMRI comparison. Neuroimage 50, 1589-1598. doi: 10.1016/j.neuroimage.2009.12.060

Luukinen, H., Herala, M., Koski, K., Honkanen, R., Laippala, P., and Kivela, S. L. (2000). Fracture risk associated with a fall according to type of fall among the elderly. Osteoporos. Int. 11, 631-634. doi: 10.1007/s001980070086

Maki, B. E., and McIlroy, W. E. (1997). The role of limb movements in maintaining upright stance: the "change-in-support" strategy. Phys. Ther. 77, 488-507. doi: $10.1093 / \mathrm{ptj} / 77.5 .488$

Malouin, F., Richards, C. L., Jackson, P. L., Dumas, F., and Doyon, J. (2003). Brain activations during motor imagery of locomotor-related tasks: a PET study. Hum. Brain Mapp. 19, 47-62. doi: 10.1002/hbm.10103

Marx, E., Stephan, T., Nolte, A., Deutschländer, A., Seelos, K. C., Dieterich, M., et al. (2003). Eye closure in darkness animates sensory systems. Neuroimage 19, 924-934. doi: 10.1016/S1053-8119(03)00150-2

Massion, J. (1994). Postural control system. Curr. Opin. Neurobiol. 4, 877-887. doi: 10.1016/0959-4388(94)90137-6

McNaughton, B. L., Barnes, C. A., Gerrard, J. L., Gothard, K., Jung, M. W., Knierim, J. J., et al. (1996). Deciphering the hippocampal polyglot: the hippocampus as a path integration system. J. Exp. Biol. 199(Pt 1), 173-185.

Mihara, M., Miyai, I., Hattori, N., Hatakenaka, M., Yagura, H., Kawano, T., et al. (2012). Cortical control of postural balance in patients with hemiplegic stroke. Neuroreport 23, 314-319. doi: 10.1097/WNR.0b013e328351757b

Mochizuki, G., Sibley, K. M., Cheung, H. J., Camilleri, J. M., and McIlroy, W. E. (2009). Generalizability of perturbation-evoked cortical potentials: independence from sensory, motor and overall postural state. Neurosci. Lett. 451, 40-44. doi: 10.1016/j.neulet.2008.12.020

Morganti, F., Gaggioli, A., Castelnuovo, G., Bulla, D., Vettorello, M., and Riva, G. (2003). The use of technology-supported mental imagery in neurological rehabilitation: a research protocol. Cyberpsychol. Behav. 6, 421-427. doi: 10. 1089/109493103322278817

Mulder, T. (2007). Motor imagery and action observation: cognitive tools for rehabilitation. J. Neural Transm. 114, 1265-1278. doi: 10.1007/s00702-0070763-z

Munzert, J., Zentgraf, K., Stark, R., and Vaitl, D. (2008). Neural activation in cognitive motor processes: comparing motor imagery and observation of gymnastic movements. Exp. Brain Res. 188, 437-444. doi: 10.1007/s00221-0081376-y 
Nadeau, S. E. (2007). Gait apraxia: further clues to localization. Eur. Neurol. 58, 142-145. doi: 10.1159/000104714

Naito, E., and Hirose, S. (2014). Efficient foot motor control by Neymar's brain. Front. Hum. Neurosci. 8:594. doi: 10.3389/fnhum.2014.00594

Nashner, L. M. (1976). Adapting reflexes controlling the human posture. Exp. Brain Res. 26, 59-72. doi: 10.1007/BF00235249

Nashner, L. M. (1980). Balance adjustments of humans perturbed while walking. J. Neurophysiol. 44, 650-664. doi: 10.1152/jn.1980.44.4.650

Orr, E. L., Lacourse, M. G., Cohen, M. J., and Cramer, S. C. (2008). Cortical activation during executed, imagined, and observed foot movements. Neuroreport 19, 625-630. doi: 10.1097/WNR.0b013e3282fbf9e0

Pai, Y. C., and Bhatt, T. S. (2007). Repeated-slip training: an emerging paradigm for prevention of slip-related falls among older adults. Phys. Ther. 87, 1478-1491. doi: 10.2522/ptj.20060326

Patel, J., Qiu, Q., Yarossi, M., Merians, A., Massood, S., Tunik, E., et al. (2017). Exploring the impact of visual and movement based priming on a motor intervention in the acute phase post-stroke in persons with severe hemiparesis of the upper extremity. Disabil. Rehabil. 39, 1515-1523. doi: 10.1080/09638288. 2016.1226419

Pavol, M. J., and Pai, Y. C. (2007). Deficient limb support is a major contributor to age differences in falling. J. Biomech. 40, 1318-1325. doi: 10.1016/j.jbiomech. 2006.05.016

Pavol, M. J., Runtz, E. F., and Pai, Y. C. (2004). Diminished stepping responses lead to a fall following a novel slip induced during a sit-to-stand. Gait Posture 20, 154-162. doi: 10.1016/j.gaitpost.2003.08.004

Petersen, T. H., Willerslev-Olsen, M., Conway, B. A., and Nielsen, J. B. (2012). The motor cortex drives the muscles during walking in human subjects. J. Physiol. 590, 2443-2452. doi: 10.1113/jphysiol.2012.227397

Picard, N., Matsuzaka, Y., and Strick, P. L. (2013). Extended practice of a motor skill is associated with reduced metabolic activity in M1. Nat. Neurosci. 16, 1340-1347. doi: 10.1038/nn.3477

Pisotta, I., and Molinari, M. (2014). Cerebellar contribution to feedforward control of locomotion. Front. Hum. Neurosci. 8:475. doi: 10.3389/fnhum.2014.00475

Porro, C. A., Francescato, M. P., Cettolo, V., Diamond, M. E., Baraldi, P., Zuiani, C., et al. (1996). Primary motor and sensory cortex activation during motor performance and motor imagery: a functional magnetic resonance imaging study. J. Neurosci. 16, 7688-7698. doi: 10.1523/JNEUROSCI.16-23-07688.1996

Quant, S., Adkin, A. L., Staines, W. R., Maki, B. E., and McIlroy, W. E. (2004). The effect of a concurrent cognitive task on cortical potentials evoked by unpredictable balance perturbations. BMC Neurosci. 5:18. doi: 10.1186/14712202-5-18

Rankin, J. K., Woollacott, M. H., Shumway-Cook, A., and Brown, L. A. (2000). Cognitive influence on postural stability: a neuromuscular analysis in young and older adults. J. Gerontol. A Biol. Sci. Med. Sci. 55, M112-M119. doi: 10. 1093/gerona/55.3.M112

Saimpont, A., Lafleur, M. F., Malouin, F., Richards, C. L., Doyon, J., and Jackson, P. L. (2013). The comparison between motor imagery and verbal rehearsal on the learning of sequential movements. Front. Hum. Neurosci. 7:773. doi: 10.3389/fnhum.2013.00773

Sharp, D. J., Bonnelle, V., De Boissezon, X., Beckmann, C. F., James, S. G., Patel, M. C., et al. (2010). Distinct frontal systems for response inhibition, attentional capture, and error processing. Proc. Natl. Acad. Sci. U.S.A. 107, 6106-6111. doi: 10.1073/pnas.1000175107

Shumway-Cook, A., and Woollacott, M. H. (2007). Motor Control: Translating Research into Clinical Practice. Philadelphia, PA: Lippincott Williams \& Wilkins.

Singh-Curry, V., and Husain, M. (2009). The functional role of the inferior parietal lobe in the dorsal and ventral stream dichotomy. Neuropsychologia 47, 1434-1448. doi: 10.1016/j.neuropsychologia.2008.11.033
Sipp, A. R., Gwin, J. T., Makeig, S., and Ferris, D. P. (2013). Loss of balance during balance beam walking elicits a multifocal theta band electrocortical response. J. Neurophysiol. 110, 2050-2060. doi: 10.1152/jn.00744.2012

Sterzer, P., and Kleinschmidt, A. (2010). Anterior insula activations in perceptual paradigms: often observed but barely understood. Brain Struct. Funct. 214, 611-622. doi: 10.1007/s00429-010-0252-2

Tang, P.-F., Woollacott, M. H., and Chong, R. K. (1998). Control of reactive balance adjustments in perturbed human walking: roles of proximal and distal postural muscle activity. Exp. Brain Res. 119, 141-152. doi: 10.1007/s00221005 0327

Taube, W., Mouthon, M., Leukel, C., Hoogewoud, H. M., Annoni, J. M., and Keller, M. (2015). Brain activity during observation and motor imagery of different balance tasks: an fMRI study. Cortex 64, 102-114. doi: 10.1016/j. cortex.2014.09.022

van der Meulen, M., Allali, G., Rieger, S. W., Assal, F., and Vuilleumier, P. (2014). The influence of individual motor imagery ability on cerebral recruitment during gait imagery. Hum. Brain Mapp. 35, 455-470. doi: 10.1002/hbm. 22192

Wang, J., Wai, Y., Weng, Y., Ng, K., Huang, Y. Z., Ying, L., et al. (2009). Functional MRI in the assessment of cortical activation during gait-related imaginary tasks. J. Neural Transm. 116, 1087-1092. doi: 10.1007/s00702-009$0269-\mathrm{y}$

Woollacott, M. H., and Tang, P. F. (1997). Balance control during walking in the older adult: research and its implications. Phys. Ther. 77, 646-660. doi: $10.1093 / \mathrm{ptj} / 77.6 .646$

Yang, F., Bhatt, T., and Pai, Y. -C. (2009). Role of stability and limb support in recovery against a fall following a novel slip induced in different daily activities. J. Biomech. 42, 1903-1908. doi: 10.1016/j.jbiomech.2009.05.009

Yang, F., Bhatt, T., and Pai, Y.-C. (2011). Limits of recovery against slip-induced falls while walking. J. Biomech. 44, 2607-2613. doi: 10.1016/j.jbiomech.2011.08. 018

Yang, Y. L., Deng, H. X., Xing, G. Y., Xia, X. L., and Li, H. F. (2015). Brain functional network connectivity based on a visual task: visual information processingrelated brain regions are significantly activated in the task state. Neural Regen. Res. 10, 298-307. doi: 10.4103/1673-5374.152386

Zehr, E. P., Komiyama, T., and Stein, R. B. (1997). Cutaneous reflexes during human gait: electromyographic and kinematic responses to electrical stimulation. J. Neurophysiol. 77, 3311-3325. doi: 10.1152/jn.1997.77.6.3311

Zhu, M.-H., Wang, J., Gu, X.-D., Shi, M.-F., Zeng, M., Wang, C.-Y., et al. (2015). Effect of action observation therapy on daily activities and motor recovery in stroke patients. Int. J. Nurs. Sci. 2, 279-282. doi: 10.1016/j.jstrokecerebrovasdis. 2015.02.022

Zwergal, A., Strupp, M., Brandt, T., and Buttner-Ennever, J. A. (2009). Parallel ascending vestibular pathways: anatomical localization and functional specialization. Ann. N. Y. Acad. Sci. 1164, 51-59. doi: 10.1111/j.1749-6632.2009. 04461.x

Conflict of Interest Statement: The authors declare that the research was conducted in the absence of any commercial or financial relationships that could be construed as a potential conflict of interest.

Copyright (๑ 2018 Bhatt, Patel, Dusane, DelDonno and Langenecker. This is an open-access article distributed under the terms of the Creative Commons Attribution License (CC BY). The use, distribution or reproduction in other forums is permitted, provided the original author(s) and the copyright owner(s) are credited and that the original publication in this journal is cited, in accordance with accepted academic practice. No use, distribution or reproduction is permitted which does not comply with these terms. 